\title{
ORT_02 - Microbial profile of intermediate process solutions identified by bioburden test in a pharmaceutical industry
}

Luciana Veloso da Costa ${ }^{1}$; Bruna de Almeida do Vale ${ }^{1}$; Cristhiane Falavina dos Reis ${ }^{1}$; Joyce Modesto de Andrade $^{1}$; Josiane Machado Vieira Mattoso ${ }^{1}$; Igor Barbosa da Silva ${ }^{1}$; Marcelo Luiz Lima Brandão ${ }^{1}$

${ }^{1}$ Fiocruz/Bio-Manguinhos.

Introduction: Bioburden monitoring is essential to contamination control of pharmaceutical products. Microbiological load may represent a potential risk for patients if the sterilization process is not effective and/or due to the presence of allergens or toxins. Although bioburden can be eliminated by terminal sterilization or filtration processes, it is important to monitor it before final processing, regarding the amount and species of microorganisms that are present.

Objective: To evaluate the microorganism's species isolated in intermediate process solutions samples to be used in immunobiologicals formulations from a pharmaceutical industry before sterilizing by filtration.

Methodology: All the microorganisms isolated from the bioburden test in the period from January 2018 to December 2020 were evaluated. They were classified into three groups according to the process criticality: Group 1- critical microorganisms listed in the Brazilian and International Pharmacopoeias (Escherichia coli, Pseudomonas aeruginosa, Salmonella, Staphylococcus aureus and Candida albicans); Group 2- questionable microorganisms that can be a risk to the process; Group 3- non-questionable microorganisms, that are not a risk to the process. The Group 2 microorganisms were evaluated according to the following criteria: indication of fecal contamination, pathogenicity for humans, and production of toxins that can be perpetuated throughout the process with risk to patients. Microorganisms identified as human pathogens have been classified into three categories: 1)pathogens that can cause infections in immunocompetent individuals; 2)opportunists that can show virulence and are responsible for infections in immunocompromised or debilitated individuals; 3)exceptional opportunists that rarely cause infections in humans.

Results: 78 generas and 171 species were isolated: two species (1.2\%) were classified in Group 1, 135 (78.9\%) in Group 2, and 34 (19.9\%) in Group 3. The two Group 1 isolated species were S.aureus and P.aeruginosa. Among the 135 Group 2 species, $92.6 \%(n=125)$ were bacteria and $7.4 \%(n=10)$ were yeasts and molds. Thirteen species $(9.6 \%)$ were fecal contamination indicators. One hundred and thirty-four $(99.3 \%)$ species were identified as human pathogens, but $4.5 \%(n=6)$ belonged to category $1 ; 38.8 \%(n=52)$ to category 2 and $56.7 \%(n=76)$ to category 3 . Twenty-eight species $(20.7 \%)$ were known for producing various toxins; and $40.7 \%(n=55)$ were endotoxins producers. In Group 3, there were $97.1 \%(n=33)$ bacteria, mainly identified in Order Caryophanales, and 2.9\% $(\mathrm{n}=1)$ fungi identified as Lecanicillium coprophilum. In the case of Group 1 bacteria's, the intermediate process solution was immediately discarded and an investigation was initiated to identify the root cause and subsequently the adoption of corrective and preventive actions. In the case of Group 2 microorganisms, a risk assessment for each specific process was carried out in order to decide whether this risk could be mitigated or eliminated along the production chain, so as not to pose patients at risk.

Conclusion: The vast majority ( $\sim 80 \%)$ of the bioburden species were questionable microorganisms.

Keywords: bioburden; pharmaceutical industry; questionable microrganisms 